Diánoia, vol. 25, no. 25, 1979

\title{
NOTAS SOBRE LA INTERVENCIÓN \\ DE LA FILOSOFÍA Y LA TEOLOGIA \\ EN EL DESARROLLO DEL CONCEPTO \\ DE FUERZA DURANTE EL SIGLO XVII
}

En este trabajo examinaré algunos aspectos del concepto de fuerza -entendida como causa del movimiento- durante el siglo xvir.

Descartes afirmó que la fuerza de un cuerpo se mide por el producto de su tamaño por su velocidad ( $t v)$; Leibniz sostuvo que se mide por el producto de la masa por la velocidad al cuadrado $\left(m v^{2}\right)$, y Newton, por su parte, la midió por el incremento del producto de la masa y la velocidad vectorial $(\Delta \overrightarrow{m v})$.

Se podria pensar que el debate entre estas tres posiciones es sólo verbal, ya que cada una de estas cantidades es aplicable a distintos tipos de problemas físicos. Intentaré mostrar que esta idea no es correcta ya que, en el fondo, dicho debate versa sobre la naturaleza de la realidad.

Este debate se dio, de hecho, en la llamada controversia sobre la vis viva, en la que Newton y los cartesianos atacaron la tesis de Leibniz, mientras que J. Bernoulli y W. S'Gravesand apoyaron a este último. En el siglo xviII, D'Alembert (quien, se considera, resolvió la controversia sobre la vis viva) sostuvo que era sólo un debate verbal. Considero que la tesis de D'Alembert es incorrecta, pues lo que se está discutiendo no es sólo cuál es la medida correcta de la fuerza motriz, sino la relación que esta fuerza guarda con la conservación o no-conservación del universo. ${ }^{1}$

El objetivo básico de este ensayo será, así, mostrar cómo las presuposiciones de estos tres autores acerca de la conservación o la no-conservación del universo los llevan a medir de distinta manera la fuerza que un cuerpo ejerce al actuar sobre otro. También intentaré mostrar cómo este presupuesto sobre la conservación o no-conservación del universo depende de una concepción específica de Dios, el creador de este universo, o sea, que la controversia sobre la vis viva no sólo fue un debate físico, sino también un debate metafísico. Más generalmente, intentaré mostrar que, durante el siglo xvir, el problema de la fuerza se intentó resolver apelando a consideraciones no sólo físicas, sino también filosófico-teológicas.

Descartes sostuvo una concepción mecanicista del mundo físico, esto es, sostuvo que está constituido por, y por ende es explicable en términos de, materia y movimiento. Con respecto a la materia, consideró que su esencia

1 Es importante notar que para los pensadores del siglo xvIr, a diferencia de los anteriores, la cuestión de si el universo es estable o se conserva, se plantea específicamente en términos de la conservación de entidades cuantificables. 
o "atributo" es la extensión o tridimensionalidad, o sea, que la materia es idéntica al espacio (Pr. II, 10-13; [4], pp. 41-43); lo cual implica que los objetos materiales sólo tienen tamaño, figura y son sujetos de movimiento o reposo. La tesis de que la materia es idéntica al espacio es el resultado del intento teórico cartesiano de comprender en qué consiste la matematización de la física: consiste en la reducción de la física a la matemática (más precisamente, a la geometría), y dicha reducción es posible, nos dice Descartes, porque la materia no es más que espacio. También sostuvo que la materia es pasiva o inerte en el sentido de que no tiene actividad: no puede continuar exisstiendo por sí misma (sino que requiere del concurso de Dios), ni puede ser causa de su propio movimiento (M. II; [3], p. 151).

Con respecto al movimiento, Descartes consideró que era un "modo", estado o propiedad inherente de la materia (Pr. II, 36; [4], pp. 53-54), ya que él trabajaba con una ontología planteada en términos de atributo y modo. Sin embargo, también sostuvo que el movimiento es un principio de diversificación de la materia (Pr. II, 23; [4], p. 47) y que el movimiento opera como causa o fuerza en el impacto ( $c f$. más adelante), lo cual implica que el movimiento es un agente activo que actúa sobre la materia -idea que, obviamente, contradice su tesis de que el movimiento es un modo de la substancia pasiva.

De acuerdo con su programa racionalista, Descartes intentó derivar, de razonamientos puramente a priori, las leyes fundamentales de la naturaleza. La presuposición de estos razonamientos es que el mundo material debe ser de cierta manera dado que fue creado por un Dios que tiene ciertas caracteristicas. Específicamente, Descartes sostuvo que: 1) Dios creó la materia, y también creó una cantidad determinada de movimiento que distribuyó no uniformemente a través de la materia; 2) dado que Dios es inmutable, t́l conserva la materia y la cantidad total de movimiento que śl creó (Pr. II, 36; [4], p. 54). La manera en que la cantidad total de movimiento en el universo se conserva es la siguiente: a) Mientras un cuerpo material (o sea, una parte de la materia) no interactúe con otro, conservará su cantidad de movimiento o "momento" (tv), es decir, continuará en su estado de reposo o de movimiento uniforme rectilineo - principio de inercia, o primera y segunda leyes de la naturaleza cartesianas.

La primera ley dice:

Y por esta misma inmutabilidad de Dios, pueden conocerse ciertas reglas o leyes de la naturaleza... La primera de éstas es que toda cosa, en tanto que simple e indivisa, queda, por lo que de ella depende, siempre en el mismo estado, y nunca puede cambiarse sino por causas externas. Así, si alguna parte de la materia es cuadrada, fácilmente nos persuadiremos que perpetuamente seguirá siendo cuadrada, si no adviene algo de otra parte que cambie su forma. Si descansa, no creemos que empezará nunca a moverse, a no ser que sea impulsada a ello por alguna causa. $Y$ si se mueve, no hay más razón para que creamos que la misma espontáneamente y no impedida por alguna otra cosa, habrá de interrum. 
pir ese movimiento suyo. Y por eso ha de concluirse que lo que se mueve, en lo que de él dependa, se mueve siempre (Pr. II, 37; [4], p. 54)."2

La segunda ley de la naturaleza dice lo siguiente:

Cada parte de la materia, mirada por separado, nunca tiende a seguirse moviendo según ninguna línea curva, sino sólo según líneas rectas (Pr. II, $39 ;[4]$, p. 55).

b) En el caso de que los cuerpos interactúen, lo cual sólo sucede por contacto (M. II; [3], p. 151) o impacto, también se conserva la cantidad de movimiento porque, al mover un cuerpo a otro, pierde tanto movimiento como le da al otro - tercera ley cartesiana de la naturaleza. Textualmente:

Yo sostengo que hay una cierta cantidad de movimiento en toda la materia creada que nunca aumenta ni disminuye, de tal forma que cuando un cuerpo mueve a otro, pierde tanto de su movimiento como le da al otro (cit. en [10], p. 69). ${ }^{3}$

Es de notarse que, como para Descartes la naturaleza sólo está constituida de materia y movimiento, el principio de inercia, que especifica la conducta de un cuerpo material cuando éste no interactúa con otros, y el principio de impacto, que especifica lo que sucede en la interacción entre cuerpos, son los dos únicos principios o leyes que él necesita para fundar la ciencia de la naturaleza.

Hemos visto así que Descartes deriva las tres leyes fundamentales de la naturaleza a partir de la inmulabilidad de Dios, esto es, del hecho de que Dios conserva el mundo como es en el momento en que lo conserva. Por tanto, podríamos extender la tesis de Koyré de que "la ley suprema de El Mundo (libro sobre física que Descartes nunca publicó) es la ley de la constancia o conservación" ([10], p. 71) a la tesis de que la ley suprema de la naturaleza es, para Descartes, la ley de la conservación. Este principio resulta muy poderoso en las manos de Descartes, ya que a partir de él (junto con algunas asunciones auxiliares) deriva, como vimos, las tres leyes fundamentales de la naturaleza.

Examinemos en detalle la ley de impacto o tercera ley de la naturaleza, teniendo en cuenta que por cantidad de movimiento Descartes entiende el producto del tamaño y la velocidad (escalar) de un cuerpo (Pr. II, 36; [4], p. 53; y Pr. II, 41; [4], p. 57). Reza la ley:

Cuando un cuerpo en movimiento se encuentra [choca] con otro... si

2 Nótese que en esta ley Descartes argumenta que la permanencia del movimiento se debe a que éste es un modo o estado de la materia.

3 Cf. también el Pr. 11, 42; [4], p. 57, donde Descartes dice que la conservación de la cantidad de movimiento en el impacto depende de la inmutabilidad de Dios. 
tiene más [fuerza para seguir en línea recta que el otro para resistirle], entonces mueve consigo al otro cuerpo y pierde tanto movimiento cuanto le da del suyo (Pr. II, 40; [4], p. 56).

Podemos ahora preguntarnos qué sucede en el impacto según Descartes. Él nos dice que:

cuando Dios creó el mundo, El debió no sólo haber asignado varios movimientos a sus varias partes, sino también debió haber causado sus impulsos mutuos y la transferencia del movimiento de unos a otros; y dado que Él ahora preserva el movimiento por la misma actividad y de acuerdo a las mismas leyes, que cuando Él lo creó, Él no lo preserva como una propiedad inherente constante de partes dadas de la materia, sino como algo que pasa de una parte a otra cuando chocan (Pr. II 42; [2], p. 219).

Sin embargo, como Descartes nota, el pensar que lo que sucede en el impacto es que una cierta cantidad de movimiento se trasmite o transfiere a otro pedazo de materia implica que el movimiento no es una propiedad inherente de los cuerpos, es decir, que no es un modo. La razón por la cual Descartes parece haber sostenido esta concepción del impacto, como transferencia de movimiento, a pesar de que contradice su tesis de que el movimiento es un modo, es que es una manera simple y clara de explicar cómo la cantidad total de movimiento que Dios creó se conserva en la interacción entre cuerpos.

Descartes también desarrolló otra concepción de impacto que apela a fuerzas. Como vimos anteriormente (Pr. II, 40; cf. arriba) Descartes enuncia su tercera ley en términos de fuerzas o poderes de acción o resistencia. Más adelante nos dice:

Pero aquí ha de advertirse con cuidado en qué consiste la fuerza de cada cuerpo para actuar sobre otro o para resistir la acción de otro. Precisamente en esto: en que cada cosa tiende a permanecer, en lo que de ella dependa, en el mismo estado en que se halla, según la ley formulada en primer término [i. e. la primera ley de la naturaleza]. De aquí, pues, que lo que está unido a otra cosa tiene alguna fuerza para impedir su desunión...; lo que está quieto, para permanecer en su quietud, y para resistir por consiguiente a todas aquellas cosas que pueden cambiarla; lo que se mueve, para perseverar en su movimiento, esto es, en el movimiento de igual celeridad y dirección (Pr. II, 43; [4], pp. 57-58).

Lo que Descartes sostiene aquí es que, de acuerdo con el principio de inercia, un cuerpo tiene un poder de permanecer en descanso, o de permanecer moviéndose uniforme y rectilíneamente, y que este poder de perseverar en un estado es idéntico a las fuerzas de acción o reacción que cualquier cuerpo ejerce en el impacto ([5], pp. 25, 37, 50,62, 65).

La fuerza de acción o resistencia que un cuerpo en movimiento tiene, es, 
pues, una propiedad inherente, un modo del cuerpo, y el cuerpo, por tanto, lo tiene, ya sea que interactúe o no con otros. Esta fuerza se mide por el producto del tamaño y la velocidad (Pr. II, 36; [4], p. 53; y Pr. II, 43; [4], p. 58). En cambio, en el caso de los cuerpos en reposo, por no tener velocidad, la fuerza de resistencia debe medirse por el producto del tamaño del cuerpo en reposo y la velocidad del cuerpo que choca contra él:

La razón por la que sostengo que un cuerpo sin movimiento nunca puede ser movido por otro que sea más pequeño, cualquiera que sea su velocidad, es que es una ley de la naturaleza que un cuerpo que mueve a otro debe tener más fuerza para moverlo que el otro tiene fuerza para resistirlo. Pero este exceso sólo puede depender de su tamaño; pues el cuerpo que no tiene movimiento, tiene tantos grados de resistencia como el otro, que se mueve, tiene de velocidad (cit. en [5], p. 26).

(Nótese que esta fuerza de reposo no es una propiedad inherente del cuerpo, esto es, algo que un cuerpo tenga independientemente de sus relaciones con otros cuerpos, ya que la fuerza de descanso de un cuerpo varía de acuerdo con las diferentes velocidades que los cuerpos que chocan contra él puedan tener.)

Para Descartes, las fuerzas actúan en el impacto de la siguiente manera (interpretación de Gabbney ([5], pp. 16 y 21), que sigue a la de Herivel, The Background to Newton's Principia). El impacto es una competencia entre tuerzas desiguales que se oponen. Lo que esto significa es que un problema de impacto se resuelve comparando la fuerza motriz de un cuerpo y la fuerza de resistencia del otro, para calcular el exceso, del cual dependerá el retardamiento del cuerpo que actúa o la aceleración del cuerpo recipiente. $O$ sea, lo que sucede en el impacto no es una transferencia de movimiento sino que la fuerza motriz de $A$ causa que $B$ (que tiene menos fuerza que $A$ ) adquiera más movimiento; de hecho, tanto movimiento (velocidad) como $A$ pierde. Es importante notar que la fuerza de movimiento o la fuerza de descanso pueden ser conocidas antes de que los dos cuerpos interactúen, por lo que el resultado de un impacto puede determinarse por adelantado.

Para resumir, la doctrina cartesiana acerca de la operación de las fuerzas en el impacto consiste en las siguientes dos tesis: a) la fuerza es un poder intrínseco que un cuerpo tiene, ya sea para mantener su estado o para resistir la causa externa que busca cambiar su estado; $b$ ) el resultado de una colisión se determina por el concurso entre fuerzas desiguales opuestas y el principio de conservación de la cantidad de movimiento.

Podemos ahora preguntarnos si Descartes puede tener un concepto de fuerza, dada su concepción del universo. Para esto, determinemos primero si Descartes puede hablar de una fuerza que mantenga al cuerpo en su estado de movimiento inercial o reposo, 4 ya que si no puede hablar de fuerzas de

4 Esto lo sugiere Descartes en algunas versiones del principio de inercia, por ejemplo, "es cierto que del mero hecho de que un cuerpo ha comenzado a moverse, se sigue que 
perseverancia en un estado, tampoco podrá hablar de fuerzas de resistencia o acción.

Demos una definición de fuerza: fuerza es una propiedad inherente del cuerpo, así como la figura, el tamaño, etcétera; la fuerza no es reductible o explicable en términos de otras propiedades; la fuerza es una causa del movimiento, descanso, resistencia o acción. Ahora bien, considero que definido así el concepto de fuerza, Descartes no puede tener este concepto, por dos razones principales. En primer lugar, como sostiene Koyré ([10], pp. 66-67 y 69), Descartes introdujo la noción de un estado de movimiento o descanso. (i. e. la noción de que son modos) precisamente para indicar que el movimiento no es un proceso (como para Aristóteles y la teoría del impetus), sino una condición en que se hallan los cuerpos, y que por tanto no se requiere una causa o fuerza que explique por qué los cuerpos continúan en su estado de movimiento o descanso. En segundo lugar, para Descartes la materia es totalmente pasiva $y$, por ende, no puede tener una fuerza interna: en este caso sería activa, ya que tendría el poder de continuar moviéndose a si misma. En otros términos, si la esencia de la materia es extensión, es decir, si los cuerpos sólo pueden tener propiedades espaciales, ențonces la materia no puede tener poderes, tendencias o fuerzas, ya que éstas no son propiedades espaciales.

Como vimos anteriormente, Descartes sostiene que las fuerzas de resistencia o acción, que explican el comportamiento de los cuerpos en el impacto, son idénticas a las fuerzas de perseverancia en un estado. Pero dado que Descartes no puede sostener que hay fuerzas en el sentido de una propiedad inherente irreductible en los cuerpos que causa la perseverancia en un estado, parece ser que él solamente puede fundar las fuerzas de resistencia o acción en la mera extensión y en el hecho irreductible de que los cuerpos mantienen sus estados a menos de que una causa externa los modifique. Algunos pasajes (por ejemplo, Pr. II, 43, citado en la p. 90) sugieren que tal era su intención. Ahora bien, Leibniz consideró que Descartes sostenía esto, es decir, que las fuerzas de resistencia están basadas en la mera perseverancia en el estado, y ofreció la siguiente crítica:

Yo admito que cada cosa singular persevera en su propio estado hasta que hay alguna razón que la haga cambiar - un principio de necesidad metatísica. Pero una cosa es perseverar en un estado dado hasta que algo ocasione un cambio; otra cosa, y aún más importante, es no ser indiferente y tener una fuerza o inclinación, por así decir, para mantener un estado dado y, con ello, ofrecer resistencia a una causa del cambio. ([14], p. 461)... Si $B$ [que está en reposo] fuese indiferente al movimiento o reposo, se dejaria empujar por $A$ sin resistirlo y sin disminuir la velocidad o cambiar la dirección de $A$ ([12], p. 100).

O sea, para Leibniz, a) un cuerpo mantiene su estado por ser indiferente al movimiento o al reposo, y es indiferente porque carece de todo poder o tiene en él una fuerza para continuar moviéndose; y también, del mero hecho de que se ha parado en un cierto lugar, tiene la fuerza para continuar all" (cit. en [5], p. 24). 
fuerza (idea clásica de Galileo); y $b$ ) si un cuerpo es indiferente al movimiento o descanso, entonces no puede oponer una fuerza de resistencia a otro cuerpo durante el impacto. Así, lo que Leibniz sostiene es que del hecho de que un cuerpo mantenga su estado no se sigue que opondrá resistencia al cambio de estado, ya que un cuerpò mantiene su estado sólo porque es. indiferente al estado en que se encuentra.

Hemos visto asi que la doctrina de Descartes acerca del impacto, que es, según él, el único caso de interacción entre cuerpos, presenta serias dificultades. Por una parte, concebir que lo que pasa durante el impacto es la trasmisión de movimiento contradice la tesis cartesiana de que el movimiento es un modo o propiedad inherente de los cuerpos, además de que aún resta por explicar el mecanismo que causa que el movimiento se trasmita. Por otra parte, Descartes no puede suponer que hay fuerzas que actúan durante el impacto, ya que esto contradice su tesis de que la materia es inerte y está caracterizada sólo por propiedades espaciales. Dada esta grave deficiencia, otros cartesianos o semicartesianos desarrollaron concepciones distintas de impacto.

Malebranche, tomando muy en serio la tesis cartesiana de que Dios no sólo crea al mundo material sino que lo conserva en su existencia porque éste ni siquiera tiene un poder de autosubsistencia, y también la tesis de que los cuerpos son pasivos y carecen de cualquier tipo de fuerza o poder, sostuvo que Dios es el único agente causal en el universo, de tal modo que lo que parece ser un caso de causalidad, tal como un cambio de movimiento debido al impacto, no es más que la ocasión para la intervención causal directa de Dios.

Por otra parte, nos dice Malebranche, no hay buenas razones para creer que las fuerzas existen. Por un lado, las fuerzas se postulan para explicar cambios de estado, pero como las fuerzas no pueden observarse, no hay ninguna prueba satisfactoria de que existan ([7], p. 206). Por otro lado, podemos explicar el impacto sin apelar a fuerzas: lo que sucede cuando dos cuerpos chocan es que su impenetrabilidad (que es deducible de la extensión) causa que cambien su estado - o más bien, es la ocasión para la intervención causal divina ([7], p. 199). Así pues, Malebranche rechaza la existencia de fuerzas por razones metafísicas - Dios es el único agente causal-, por razones epistemológicas - las fuerzas no son observables - y por razones físicas -el impacto puede explicarse apelando a la impenetrabilidad de los cuerpos.

Leibniz, un semicartesiano, sostuvo que la extensión (que implica sólo la indiferencia de la materia al movimiento o reposo) no es suficiente para explicar por qué los cuerpos resisten el cambio de estado. A partir de esto concluyó que la substancia (llamada por él "mónada") debe caracterizarse, no por la extensión, sino por la fuerza o actividad. Leibniz dio razones no sólo físicas, sino también teológico-filosóficas para sostener que la esencia de la substancia es la fuerza: "las viejas formas o entelequias no son sino fuerzas. De esa manera [es decir, considerando que la esencia de las substancias es la fuerza] creo en rehabilitar la filosofia antigua o escolástica, que es tan útil a la teología, sin eliminar ninguno de los descubrimientos modernos o expli- 
caciones mecánicas, ya que la mecánica misma presupone la consideración de las fuerzas" ([12], pp. 104-105).

La tesis de que la substancia debe caracterizarse por la fuerza no fue meramente una tesis metafisica que Leibniz opuso a la concepción geométrica cartesiana de la materia, sino que también la utilizó para desarrollar una teoría física distinta a la cartesiana. Recordemos que para Descartes el universo se conserva porque la cantidad total de movimiento se conserva, tanto en el caso de que los cuerpos no interactúen como en el caso de que interactúen por medio del impacto. Ahora bien, Leibniz estaba de acuerdo en que el uni: verso se conserva, pero no estaba de acuerdo en que la conservación del universo se da a través de la conservación de la cantidad de movimiento. Leibniz aceptó la prueba experimental de Wallis, Wren y Huygens de que lo que se conserva en el impacto es el producto de la masa (o peso o tamaño; en este tiempo aún había dudas acerca de cómo se mide correctamente la cantidad de materia) y la velocidad vectorial de los cuerpos que chocan. Una vez descubierto que lo que se conserva en el impacto es $m \vec{v}$, resultó claro que, en el impacto entre cuerpos no elásticos, el movimiento (i. e. la velocidad escalar) no se conserva ([8], p. 236; [9], p. 23). Por ejemplo, supongamos que dos cuerpos no elásticos con la misma masa $\left(m_{1}=m_{2}\right)$ viajan a la misma velocidad escalar y con direcciones opuestas $\left(\vec{v}_{1}=-\vec{v}_{2}\right)$; dado que la cantidad total de movimiento de los dos cuerpos es igual a cero

$$
\left(m_{1} \vec{v}_{1}+m_{2} \vec{v}_{2}=m_{1} \vec{v}_{1}+m_{1}\left(-\overrightarrow{v_{1}}\right)=m_{1} \vec{v}_{1}-m_{1} \vec{v}_{1}=0\right)
$$

eso significa que al chocar dejarán de moverse por completo (ya que la cantidad de movimiento, que es igual a cero, se conserva). Dado que sabía que el principio de conservación de la cantidad de movimiento no implica la conservación del movimiento, Leibniz consideró que el elemento fundamental de la naturaleza que debe conservarse, para que todo el universo se conserve ([6], p. 281; [8], p. 237), no puede ser la cantidad de movimiento. Hemos visto que por razones filosóficas y científicas Leibniz creía que el elemento fundamental de la naturaleza es la fuerza; así, pensó que lo que debía conservarse era la fuerza, y por tanto buscó una manera de medir la fuerza tal que resultara que ésta se conservara en el universo:

es razonable [pensar que] la misma suma de la fuerza motriz deba conservarse en la naturaleza y no disminuir -dado que nunca vemos que un cuerpo pierda fuerza sin que ésta sea transferida a otro cuerpo- o aumentar ([11], p. 296, cf. p. 314).

Leibniz, gracias al trabajo de Huygens, sabía que $m v^{2}$ se conserva en los impactos puramente elásticos ([9], p. 23; [16], p. 289). Así, pues, sostuvo que la medida correcta de fuerza (de la fuerza asociada con cuerpos en movimiento) era $m v^{2}$; a esta fuerza la llamó vis viva. En "Una breve demostración de un 
error notable de Descartes y otros acerca de una ley natural" [11], argumentó, utilizando el teorema galileano de la caída libre, que la medida de fuerza que se conserva es $m v^{2}$. Y puesto que la conservación de $m v^{2}$ sólo se da en impactos entre cuerpos elásticos, y puesto que Leibniz creía en el principio de continuidad según el cual ningún cambio ocurre por saltos, de tal forma que el impacto entre cuerpos no elásticos viola este principio porque en este caso los cambios de velocidad son instantáneos (i. e., hay un salto de una velocidad a otra), concluyó, a diferencia de Descartes y Newton, que la materia es un pleno elástico ([15], p. 15).

Mencionamos anteriormente que la tesis de Leibniz de que la fuerza de movimiento se mide por $m v^{2}$ generó la controversia sobre la vis viva, en la que también participaron los cartesianos y Newton. Se considera que D'Alembert (Traité de Dynamic, 1743) resolvió esta controversia sosteniendo que la disputa era sólo verbal, ya que tanto $m v$ como $m v^{2}$ miden distintos efectos ([6], pp. 282-283). ${ }^{5}$ En contra de D'Alembert, consideramos que la disputa sobre la vis viva es una disputa acerca de la conservación de la fuerza ([9], p. 22) y no sólo acerca de cómo se mide la fuerza de movimiento. Veamos.

Para Leibniz, la fuerza de movimiento debe medirse como $m v^{2}$ porque sólo midiéndola así puede sostenerse que la fuerza se conserva, y dado que la fuerza es el elemento constitutivo del universo, el universo se conserva. Por otra parte, Newton no consideraba que el universo se conserva - de hecho, sostuvo que está decayendo y que requiere de la intervención directa de Dios de tiempo en tiempo para reformarlo (Opticks, Q. 31; [13], p. 180)-, y por ello pudo dar otra medida de fuerza.

En segundo lugar, como resulta claro de la controversia entre Leibniz y Clarke -este último actuó como el vocero de Newton-, el debate acerca de si el universo se conserva o no dependía, a su vez, de una concepción de Dios. Para Leibniz Dios ante todo es omnisciente ([13], p. 11), por lo cual debió tener una razón suficiente para crear este universo y no otro; dicha razón es que este universo es más perfecto que cualquier otro $\mathrm{y}$, por tanto, este universo debe poder continuar existiendo (i. e., conservarse) por sí mismo, ya que un universo que se conserva es más perfecto que un universo que requiere de la intervención directa de Dios para continuar existiendo, o para ser reparado. En cambio, para Newton, Dios es ante todo omnipotente (i. e., es la Divina Providencia) ([13], p. 14), por lo que Dios cré́ un mundo sobre el cual siempre ejerce su poder, y una manera de ejercer su poder es reformando el mundo cuando éste decae por completo.

En tercer lugar, la controversia sobre la vis viva no se puede considerar simplemente como una disputa acerca de cómo medir correctamente la fuerza de movimiento, ya que, como vimos, sostener que $m v^{2}$ se conserva implica que el mundo es un pleno elástico, mientras que si no se acepta la conservación de $m v^{2}$, ni la conservación del movimiento, es posible sostener, como Newton, que el universo está constituido por átomos duros. Podemos concluir,

5 En la mecánica clásica se considera que $\overrightarrow{m v}$ es la medida de cantidad de movimiento; $m y^{2}$ interviene en la fórmula de trabajo (a saber, $f d=m v^{2}$ ), mientras que $f=m a$. 
por tanto, que la disputa sobre la vis viva es en el fondo una disputa acerca del carácter de la realidad, es decir, acerca de si el universo se conserva, acerca de la estructura de la materia que lo constituye, y acerca del atributo cardinal de Dios.

Veamos ahora algunos aspectos del concepto de fuerza de Newton. Newton sostuvo que "el cambio de movimiento es siempre proporcional a la fuerza motriz impresa", es decir, que la fuerza impresa, que actúa sobre un cuerpo para cambiar su estado de movimiento uniforme rectilíneo o de reposo, es proporcional al cambio de estado o, más precisamente, al cambio de momento del cuerpo sobre el cual actúa $(f=\Delta m \vec{v})([1]$, p. 128). Destaquemos dos puntos en esta definición de fuerza impresa (i. e., una fuerza que se le imprime a otro cuerpo para cambiar su estado). Por una parte, hay una clara similitud entre el concepto de fuerza de Descartes y el de Newton, ya que ambos están definidos en términos de momento o cantidad de movimiento. ${ }^{6}$ Por otra parte, hay una diferencia obvia entre las definiciones de fuerza de Descartes y de Newton. Para Descartes, la fuerza es aquello que causa que un cuerpo actúe sobre otro en una colisión y cambie con ello su estado; y dado que esta causa está asociada con el cuerpo en movimiento, la midió como $m v$ (o más precisamente, como tv). En cambio, para Newton, la fuerza no es aquello que causa que un cuerpo actúe sobre otro, sino que la fuerza que un cuerpo ejerce sobre otro es aquello que causa un cambio de estado en el otro cuerpo y, consecuentemente, $f=\Delta m \vec{v}$.

Podemos preguntarnos por qué Newton desechó la idea de que la fuerza de un cuerpo se mide por la cantidad de movimiento que éste tiene y adoptó la idea de que la fuerza que un cuerpo ejerce sobre otro se mide por el cambio en la cantidad de movimiento del cuerpo sobre el cual se actuó. Para contestar esto, veamos cómo entendió Newton la ley de la inercia. Podemos pensar que Newton consideró que la ley de la inercia afirma que lo que necesita ser explicado no es por qué los cuerpos continúan moviéndose de manera uniforme y rectilínea, o continúan en reposo, sino por qué cambian su estado:

Todo cuerpo continúa en su estado de reposo, o de movimiento uniforme en línea recta, a menos que sea obligado a cambiar ese estado por fuerzas impresas sobre él ([13], p. 160).

Y puesto que Newton tenía la convicción metafísica de que los efectos (i. e., cambios de estado) son proporcionales a sus causas (i. e., a las fuerzas), se sigue que $f=\Delta m \vec{v}$.

Ahora bien, dado que la fuerza impresa es aquello que actúa sobre un

6 Cohen ha sostenido que "ningún lector podría escapar a la conclusión de que la física de colisiones e impactos fue central para el pensamiento dinámico de Isaac Newton. Una vez más, fue Descartes quien mayor influencia tuvo. Descartes enfatizó, en sus Principia, que la fuerza de un cuerpo en movimiento se mide por el producto de su magnitud y su velocidad, lo cual fue transformado por Wallis en peso y velocidad y finalmente fue transformado por Newton en masa y velocidad" ([1], p. 136). 
cuerpo para cambiar su estado, y dado que la única cosa que puede actuar sobre un cuerpo es otro cuerpo, se sigue que la fuerza es una acción que un cuerpo ejerce sobre otro, es decir, que la fuerza es relacional. Esto significa que la fuerza no es una propiedad inherente de un cuerpo, que pueda tener aun cuando está aislado de otros cuerpos, sino que sólo está presente cuando hay interacción entre cuerpos; como dice Newton: "un cuerpo ejerce esta fuerza [vis inertiae] sólo cuando otra fuerza, impresa sobre él, intenta cambiar su condición" ([13], p. 147). Además, Newton consideró que, en la interacción entre cuerpos, cada uno de ellos ejerce una fuerza sobre el otro: "Para toda acción siempre hay una reacción opuesta e igual" ([13], p. 161).

Veamos cómo opera la fuerza en el impacto de acuerdo con Newton. Supongamos que los cuerpos $A$ y $B$, que están moviéndose, chocan. Para Newton, a diferencia de Descartes, el impacto no consiste en un concurso de fuerzas, ya que ni $A$ ni $B$ tienen por sí mismos una fuerza propia que pueda combatir la fuerza del otro cuerpo. Lo que ocurre, más bien, es que $A$ ejerce sobre $B$ una fuerza (que se mide por el cambio de momento $(\overrightarrow{m v})$ de $B$ ), y $B$ resiste en el sentido de que ejerce sobre $A$ una fuerza que es igual a la fuerza ejercida por $A$ sobre $B$, sólo que con dirección opuesta. Dado que la fuerza de resistencia que $B$ ejerce sobre $A$ es una fuerza que actúa sobre $A$ para cambiar el estado de $A$ (a menos que la contrarreste una fuerza diferente impresa en $A$ en la dirección contraria), es también una fuerza impulsora; igualmente, la fuerza impulsiva que $A$ ejerce sobre $B$ puede considerarse como una fuerza de resistencia que $A$ ejerce sobre $B$ porque es la resistencia que $A$ opone a la fuerza impulsiva de $B$. De esto se sigue que la resistencia que empíricamente percibimos cuando tratamos de mover o parar un cuerpo no se debe a que el cuerpo posea una fuerza inherente de resistencia que combate la fuerza que ejercemos sobre él, como Descartes creía, sino más bien a una fuerza "reactiva" que el cuerpo ejerce sobre nosotros ([8], p. 248).

Preguntémonos ahora qué ventajas presenta la definición newtoniana de fuerza. Claramente, si la fuerza es proporcional al cambio de momento, entonces siempre que se observe un cambio de momento o cambio de estado podemos inferir que hay una fuerza actuando y, además, cuál es la medida de esa fuerza. Por ello, la definición de Newton puede cubrir un gran campo de diferentes tipos de reacciones, o fuerzas, entre los cuales se encuentran las fuerzas que actúan (o parecen actuar) a distancia, como la gravedad. En palabras de Cohen:

Al concentrarse en la acción más que en la fuerza, en el cambio de estado o de momento más que en la causa de tal cambio, Newton fue capaz de trabajar con las atracciones y fuerzas centrípetas como si éstas tuvieran la misma realidad que la percusión (impacto) y la presión ([1], p. 137).

Por otra parte, si concibiésemos como Leibniz y Descartes, que la fuerza es una propiedad inherente de los cuerpos, y por ende la midiéramos por medio 
de otras propiedades del cuerpo en que se encuentra (y no por el cambio de estado que causa), sólo podrfamos hablar de fuerzas que causan una acción donde podemos percibir sensorialmente que hay una acción, es decir, que hay dos cuerpos en contacto -en esta categoria se encuentran el impacto, la presión y algunas fuerzas centrípetas ( $v . g$., una piedra que se mueve circularmente por estar atada a un hilo que se mueve), pero no otras, a saber, aquellas que causan una acción a distancia.

Considero que nuestro breve examen del concepto de fuerza en el siglo xvi nos permite concluir que el problema cientifico de fuerza fue resuelto apelando no sólo a consideraciones experimentales sino también a consideraciones filosófico-teológicas. Esto resulta claro por lo siguiente:

I. El dehate entre Descartes y Leibniz se dio en los siguientes términos:

a) Ambos asumen que el universo se conserva porque lo creó un Dios inmutable o un Dios omnisapiente (tesis filosófico-teológica).

b) Leibniz, i) utilizando el descubrimiento experimental de que la conservación del momento en el impacto no implica la conservación del movimiento, ii) apelando a su idea de que la fuerza de acción o reacción que se ejerce durante el impacto es irreductible a otras propiedades de la materia, iii) y teniendo en cuenta su tesis filosófico-teológica de que la esencia de la substancia es la fuerza o actividad, concluye que lo que debe conservarse en el universo es la vis viva o $m v^{2}$. EI que la fuerza se mida por $m v^{2}$, Leibniz 10 sostiene a partir de razonamientos físicos (i. e., utilizando los experimentos de Huygens, y argumentando con el teorema galileano la caída libre).

II. El debate entre Leibniz y Newton se dio en términos filosófico-teológicos, a saber, en términos de la necesidad o no de principios de conservación, necesidad que a su vez dependía de concepciones particulares de Dios (i. e., de si el atributo cardinal de Dios era la omnisapiencia o la omnipotencia).

III. Un debate básico entre Newton, por una parte, y Leibniz y Descartes, por la otra, consistía en lo siguiente. Para Leibniz y Descartes, que estaban dominados por una metafísica en términos de substancia y atributo (o propiedades), la fuerza, que es una propiedad de una substancia, debía medirse en términos de otras propiedades de la substancia que ejerce la fuerza. En cambio, Newton no estaba sujeto a una metafísica en términos de substancia y atributo, por lo cual pudo pensar que la fuerza debía medirse en términos del efecto que causaba, y no en términos de las propiedades que tenía el cuerpo que la ejercia; i. e., pudo desarrollar un concepto relacional de fuerza. Como dijimos anteriormente, el que Newton desarrollase tal concepto (i. e., $f=\Delta \overrightarrow{m v}$ ) fue de gran importancia para el desarrollo de la física, ya que permitió estudiar los casos de interacción de cuerpos a distancia. 
Lo que queremos sugerir con esto es que las concepciones filosóficas que guiaron a los pensadores en el siglo xvir en su quehacer científico influyeron, por ello mismo, de manera directa en el desarrollo de la ciencia.

IV. Un debate implícito entre Malebranche y Newton gira en torno al significado de la metodología empirista. Para Malebranche, ser empirista significa que únicamente se puede aceptar la existencia de aquellas entidades que son directamente observables. En cambio, para Newton, ser empirista consiste sólo en basar el conomiento en la experiencia, es decir, significa que el conocimiento de efectos nos permite obtener conocimiento sobre las causas, mismas que no son reducibles a los efectos, y que pueden no ser directamente observables.

Así, para Malebranche, la fuerza no existe como una entidad en el universo, sino que el nombre "fuerza" sólo designa la cantidad de movimiento. Para Newton, en cambio, "fuerza" designa una entidad en el universo, distinta a la materia, y que se mide por $\Delta \overrightarrow{m v}$.

Aunque en este trabajo nos hemos centrado en la influencia del pensamiento filosófico sobre el científico, esto no implica que no consideremos que el pensamiento científico repercutió a su vez sobre el pensamiento filosófico; en verdad, claramente en el caso de Descartes y Leibniz, el objetivo filosófico era entender y justificar metafísicamente a la física mecanicista y matematizada que se desarrollaba en la época.

Instituto de InVESTIGaciones FILOSÓficas

Elta Nathan

Universidad Nagional Autónoma de México

\section{BIBLIOGRAFIA}

[1] Cohen, I. B., "Newton's Second Law and the Concept of Force in the Principia". Texas Quarterly, vol. 10, 1967.

[2] Descartes, R., Descartes Philosophical Writings. Ed. por E. Anscombe y P. T. Geach. Thomas Nelson \& Sons, Ltd., 1969.

[3] —, The Philosophical Works of Descartes. Traducido por E. Haldane y G. R. T. Ross, vol. I, Cambridge: University Press, 1968.

[4] - Principios de la filosofía. Argentina: Editorial Losada, 1951.

[5] Gabbney, A., "Force and Inertia in Seventeenth-Gentury Dynamics", Studies in History and Philosophy of Science, vol. 2, 1971.

[6] Hankins, T. L., "Eighteenth-century Attempts to Resolve the Vis Viva Controversy", Isis, vol. 56, 1965.

[7] - "The Influence of Malebranche on the Science of Mechanics during the Eighteenth Century", Journal of the History of Ideas, vol. 28, 1967.

[8] Holton, G., Introduction to Concepts and theories in Physical Science, segunda edición. Massachussetts: Addison-Wesley Publishing Co., 1973.

[9] Iltis, C., "Leibniz and the Vis Viva Controversy", Isis, vol. 62, 1971. 
[10] Koyré, A., Newtonian Studies. The University of Chicago Press, 1965.

[11] Leibniz, G. W., Leibniz Philosophical Papers and Lettres. Ed. por L. E. Loemker. Segunda edición. Holland: D. Reidel Publishing Co., 1969.

[12] --, Leibniz Selections. Ed. por P. P. Weiner. New York: Charles Scribner's Sons, 1951.

[13] The Leibniz-Clarke Correspondence. With Extracts from Newton's PRINcIPIA and Opticks. Ed. por H. G. Alexander. New York: Barnes and Noble, 1970.

[14] Nason, J. W., "Leibniz' Attack on the Cartesian Doctrine of Extension", Journal of the History of Ideas, vol. 7, núm. 4, 1946.

[15] Scott, W. J., The Conflict Between Atomism and Conservation Theory, 16641860. New York: Neale Watson Academic Publications, Inc., 1970.

[16] Westfall, R., Force in Newton's Physics. The Science of Dynamics in the Seventeenth-Century. London: Macdonald. Nueva York: American Elsevier, 1971. 